\title{
Mediaciones instrumentales entre distintas culturas de aprendizaje musical
}

\author{
Instrumental mediations between different music learning cultures
}

\author{
Amalia CASAS-MAS \\ Universidad Autónoma de Madrid - España \\ amalia.casas@uam.es
}

Cómo citar este artículo: CASAS-MAS, Amalia (2016): «Mediaciones instrumentales entre distintas culturas de aprendizaje musical», Mediaciones Sociales, no 15, pp. 151-167. DOI: http://dx.doi.org/10.5209/MESO.54548

Recibido: 5 de septiembre de 2016.

Aceptado: 27 de octubre de 2016.

\section{RESUMEN}

En el artículo se revisan las culturas de aprendizaje musical desde una mirada psicológica y social en distintos contextos tanto de tradición oral como escrita. La construcción de conocimiento y el uso de diferentes herramientas psicológicas de aprendizaje de acuerdo a los diferentes objetivos que sirven son analizadas desde el modelo socio-histórico. Estableceremos la noción de continuo entre los polos de aprendizaje formal e informal y veremos lo que supone para el aprendizaje formal la comprensión y la ampliación de herramientas de mediación del aprendizaje informal musical en la cognición. Finalmente daremos algunas claves para facilitar el entendimiento de la cultura de aprendizaje informal y su incorporación a las instituciones educativas.

PALABRAS CLAVE: cognición encarnada, contexto formal e informal, cultura de aprendizaje, mediación instrumental, música.

\footnotetext{
ABSTRACT

The article reviews the cultures of musical learning from a psychological and social perspective in different contexts of both oral and written tradition. The construction of knowledge and the use of different psychological tools of learning according to different objectives they serve are analyzed from the socio-historical model. We establish the notion of continuum between formal and informal poles of learning. In addition, we reflect on what it means understanding and expanding medi-
} 
ation tools of informal musical learning in cognition. Finally, we also intend to facilitate the understanding of the informal musical learning and its inclusion to educational institutions.

KEYWORDS: embodied cognition, formal and informal realms, leaning culture, instrumental mediation, music.

Sumario: 1. Introducción. 2. La revalorización de los contextos de aprendizaje informales: compatibilidad entre la tradición oral y escrita. 3. Mediadores culturales y desarrollo cognitivo. 4. Mediación instrumental. 5. Cultura y aprendizaje musical. 6. Los contextos de aprendizaje en la educación musical: de los formales a los informales. 7. ¿Qué aporta la comparación de aprendizaje formal e informal en música? 8. Conclusiones. Bibliografía.

\section{INTRODUCCIÓN}

Este artículo reflexiona sobre la educación musical a partir de una comparación entre los contextos de aprendizaje formales e informales. En concreto, se cuestiona la separación entre ambos contextos para explorar las aportaciones que los contextos informales pueden hacer al aprendizaje reglado o institucional.

La mayor parte de la investigación en educación musical se centra en la observación de los procesos de enseñanza en lugares institucionales o en lo que denominaremos como culturas académicas. Estos procesos están basados en la premisa de que el aprendizaje de la música es el resultado de estar expuesto a la enseñanza musical metódica, formalmente secuenciada. Las herramientas de mediación o representaciones externas en estos ámbitos se basan también en la notación, generalmente clásica (Casas y Pozo, 2008; Gruson, 1988; Hallam, 2007; Hultberg, 2002; McPherson y Gabrielsson, 2002; Woody, 1999).

Sin embargo, durante los últimos quince años ha habido un creciente interés en la consideración de los contextos no formales e informales dentro de los contextos institucionales, para ver su aplicación a la enseñanza (Dunbar-Hall y Wemyss, 2000; Robinson, 2010; Wang y Humphreys, 2009), así como en diversas formas de aprendizaje informal de la música fuera de los entornos institucionales (Folkestad, 1998; Green, 2001/02, 2008; Shah, 2006). Esto forma parte de un cambio en el enfoque de la enseñanza al aprendizaje y, por tanto, del profesor al alumno. Pero este cambio de enfoque en la investigación no quiere decir que se produzca aún un cambio similar en los contextos educativos, tanto dentro de los propios contextos formales (comúnmente asociados a la escritura) como en los contextos orales. Nuestro propósito consiste en adentrarnos en ellos para observar semejanzas y diferencias al respecto.

Para abordar esa comparación en los contextos de aprendizaje se recurrirá al concepto de cultura de aprendizaje, entendida como el conjunto de enfoques y herra- 
mientas (representaciones, procesos, contenidos y condiciones) utilizadas para transmitir la música y que median, en un contexto dado, entre quienes enseñan y/o la hacen y quienes aprenden. Los contextos en los que el aprendizaje tiene lugar no son formales o informales debido a las herramientas que se utilizan o al tipo de música en sí, sino al conjunto de prácticas culturales que los rodean. Incurriríamos en un prejuicio si entendiésemos el aprendizaje formal de la música como sinónimo de la música clásica occidental con partitura o si limitásemos el aprendizaje informal a la música popular transmitida de oído (Folkestad, 2006). Lo que se aprende y cómo los elementos están interconectados entre sí no viene determinado por el género musical o los instrumentos.

Para cumplir este objetivo en primer lugar presentaremos las investigaciones recientes en los ámbitos educativos informales en música, por qué surge esa necesidad y cómo cuestiona la compatibilidad entre la tradición oral y la escrita. En segundo lugar nos adentraremos en el marco teórico que desde la psicología socio-histórica vygotskiana argumenta la repercusión de la mediación cultural en el desarrollo cognitivo de los individuos que conforman el grupo social. A continuación describiremos los instrumentos (herramientas y signos) de los que se vale la mediación para la construcción del conocimiento. Esto a su vez consolida la formación de diferentes culturas de aprendizaje, que definiremos partiendo de las representaciones culturales socialmente construidas y de esta manera cuestionar la separación entre los contextos formales e informales. Finalmente propondremos algunas aportaciones cruciales de los contextos educativos informales en la música, basándonos en los trabajos de sociología educativa de Green $(2001 / 02,2008)$ e investigaciones previas propias para defender vías de inclusión de manera respetuosa en el ámbito institucional.

\section{LA REVALORIZACIÓN DE LOS CONTEXTOS DE APRENDIZAJE INFORMALES: COMPATIBILIDAD ENTRE LA TRADICIÓN ORAL Y ESCRITA}

Mientras que el arte ha sido considerado como un hito de la complejidad cognitiva y el lenguaje como herramienta de comunicación, la música, al igual que otras artes, ha sufrido nuestra percepción de que es una "actividad de ocio" epifenómena, y científicamente inaccesible. Esta concepción se sustenta en una tradición que se ha fundamentado en el análisis y transmisión de los sistemas externos de representación como un fin en sí mismos, principalmente la notación, que han sido las materias usadas para la propia supervivencia, pero que excluyen de manera alarmante el cuerpo, el gesto, y con él una amplia gama de emociones, que también construyen símbolos y significados. De ahí, el auge del interés por el patrimonio inmaterial, que desde los años noventa está madurando y poniendo de relieve de nuevo las destrezas y los sentidos (Muñoz Carrión, 2008). 
Un ejemplo trascendente en nuestro país ha sido el reconocimiento del flamenco como Patrimonio Inmaterial de la Humanidad por la UNESCO (2010). Cabe mencionar también las investigaciones que vienen a señalar aquello que parecía invisible hasta el momento, como los procesos psicoemocionales y corporales que desarrollan los integrantes de esta cultura de aprendizaje musical, en los casos de tradición oral, respecto de otras de tradición escrita (Casas-Mas, Pozo y Scheuer, 2015). La recuperación del cuerpo, la emoción y los procesos comunicativos están de actualidad y tenemos un acceso a ellos privilegiado mediante el dominio de conocimiento musical.

Los trabajos procedentes de la Etnomusicología han dirigido la atención hacia la musicalidad intrínseca del ser humano (Blacking, [1976] 2006) y la necesidad de explorar diferentes tipos de prácticas de aprendizaje que nos lleven más allá de la herencia recibida del siglo XIX y de la primera mitad del XX. Las investigaciones de Vallejo (2008) con la comunidad Wagogo de Tanzania son un buen ejemplo de descripción de un grupo social en el que la música está totalmente integrada con las prácticas cotidianas de todos sus miembros sin excepción ni limitación de capacidades. En esta comunidad las formas educacionales y de transmisión del conocimiento musical se activan sin usar la explicación verbal declarativa sobre la misma, sino a partir de la práctica integrada en la vida cotidiana de una manera más holística.

En la primera década de este siglo hay un creciente número de estudios que tratan de describir los tipos de prácticas y objetivos de aprendizaje en músicas que originalmente han sido de tradición oral y que poco a poco se están viendo incorporadas al ámbito académico. Pero, en esa incorporación, no dejan de aparecer conflictos, porque los mundos psicológicos y sociales desde los que se construye la tradición oral y la escrita son distintos, aunque no incompatibles. Al contrario, la diversidad de aproximación al aprendizaje está contribuyendo a replantear las formas educativas de los ámbitos académicos, ya cuestionadas en diversas ocasiones (Kingsbury, 1988; Musumeci, 2002; Nettl, 1995). Esto está suponiendo un reto de compatibilización de distintos tipos de enfoque de aprendizaje y sensibilización, y que probablemente sea de aplicación más allá del dominio musical.

\section{MEDIADORES CULTURALES Y DESARROLLO COGNITIVO}

Para analizar las formas de adquisición de la música y la cultura necesitamos adoptar un marco teórico amplio a partir del cual poder explicar y analizar el proceso de desarrollo musical desde sus inicios en un plano ontogenético. El plano ontogenético es aquel interesado en comprender cómo el ser humano va desarrollando su aprendizaje y dominio de la música desde que nace y a lo largo de su crecimiento. Una aproximación teórica global, desde la psicología, podemos encontrarla en el modelo sociohistórico, desarrollado a partir de la obra de L. S. Vygotski. Este modelo teórico pro- 
pone unos principios generales que son aplicables en distintos ámbitos de la Psicología, especialmente en aspectos del desarrollo cognitivo e incluso de la motivación.

Este autor bielorruso propuso el estudio del desarrollo desde un punto de vista evolutivo. Dicho desarrollo debía ser analizado tanto desde la historia de las especies como desde la historia de la cultura y del individuo. En el proceso evolutivo tendría un papel muy relevante la idea de la transformación de la naturaleza mediante el uso de herramientas. Esta idea que Vygotski recoge de Engels (vid. Montero, 1989) defiende que estas herramientas, y los signos entendidos como herramientas psicológicas, constituyen instrumentos mediadores en el desarrollo para la internalización de las funciones mentales transmitidas por la sociedad y la cultura.

Vygotski estableció que lo que permite al individuo desarrollar sus procesos psicológicos superiores es su naturaleza social. La formación de los procesos psicológicos implica que, en una primera fase, las funciones mentales se desarrollan de forma externa, en la interacción con otros individuos. En una segunda fase, estas funciones mentales se van internalizando, ya que el aprendiz se apropia progresivamente de esas funciones que aparecen en su interacción con los demás (Vygotski, [1931] 2000). Esta transición de los procesos del plano interpsicológico al intrapsicológico se lleva a cabo mediante la utilización de instrumentos mediadores: herramientas y signos, entre los que ocupa un lugar determinante el lenguaje.

\section{MEDIACIÓN INSTRUMENTAL}

La mediación instrumental, es decir, el uso que hace el Homo Sapiens moderno de herramientas y técnicas artificiales, es lo que le ha permitido una adaptación radicalmente distinta al medio, sin que ello suponga modificaciones de índole biológica. Vygotski distinguió dos clases de instrumentos mediadores: las herramientas -o herramientas técnicas- y los signos -también llamados herramientas psicológicas(Vygotski y Luria, [1930] 1993). Sin embargo, este autor se centró principalmente en los signos por considerar que son los que establecen la distinción entre las funciones mentales elementales, presentes también en los primates, y las superiores (Wertsch, [1991] 1993).

Ambos tipos de herramientas podrían formar parte como subcategorías de una categoría más general, que es la actividad mediada (Vygotski, [1931] 2000), ya que los dos tipos de instrumentos, técnicos y psicológicos, cumplen una función mediadora de la actividad. Pero podemos diferenciar que la herramienta técnica se orienta hacia un uso externo y tiene como objetivo provocar cambios en el objeto de su actividad; mientras que la herramienta psicológica o signo está orientada hacia la mediación interna de los procesos psicológicos y es utilizada por el ser humano para influir psico- 
lógicamente en su conducta (Vygotski, [1931] 2000). Así, la herramienta técnica es un medio para la actividad exterior, mientras que el signo lo es para la actividad interior y, por tanto, para la formación de los procesos psicológicos superiores.

El lenguaje es el sistema de signos que ocupa el centro de la investigación en la aproximación socio-histórica. Concretando estas consideraciones en el ámbito musical, cabe plantear unas preguntas: ¿el lenguaje musical podría tener semejanzas con los signos del lenguaje verbal en el sentido vygotskiano?, ¿la notación musical podría tener un paralelismo con los signos escritos del lenguaje verbal?, ¿el instrumento musical (trombón, voz, piano...) puede cumplir la misma función de mediador y por tanto formar parte del concepto de actividad mediada, tal y como Vygotski definía otros tipos de instrumentos técnicos o herramientas? El despliegue, la utilización y la función de esas "tecnologías" musicales son una importante fuente de información de cara a comprender distintas formas de organización o culturas de aprendizaje.

Sin querer desarrollar aquí la discusión acerca de las relaciones entre lenguaje verbal y música, que excederían el propósito del artículo, sí podríamos especificar algunas similitudes y diferencias por medio del desarrollo y uso de los sistemas de representación externa. Las representaciones externas son entendidas como las "marcas" que suponen el establecimiento de una relación intencional entre el medio de representación, generalmente con cualidades visuo-espaciales o visuo-temporales y el sonido como realidad representada. El análisis de los soportes de esas representaciones externas (corporal, audio, notación, etc.) que desarrolla el ser humano y el uso que de ellas hace nos permite comprender la huella cognitiva y social (Casas-Mas, Pozo y Scheuer, 2015). Parte de esos soportes serán comentados más adelante en la comparación entre los contextos formales e informales de aprendizaje.

\section{CULTURA Y APRENDIZAJE MUSICAL}

A la hora de describir la situación entre los contextos debemos ser cuidadosos en no reducirla a una oposición entre oralidad y escritura. Lilliestam (1996) afirma que, en lugar de verla como una oposición o dicotomía, debe ser vista como un continuo donde las diferentes culturas tienen diferentes grados (y tipos) de alfabetización. Hoy en día es más pertinente hablar de estrategias orales y alfabetizadas para diferentes tipos de objetivos y que funcionan más o menos bien para diferentes propósitos. Es bien sabido que los músicos de la tradición clásica priorizan la notación en comparación con los músicos no-clásicos. Por otra parte, la idea de la práctica regular y constante es crucial para intérpretes de música clásica, pero no tiene una alta prioridad en los músicos no-clásicos, que pueden practicar de manera mucho más esporádica e intensiva, y utilizar grabaciones ( $\mathrm{mp} 3, \mathrm{mp} 4$, etc.) como herramientas cotidianas (Green, 2001/02, 2008). 
Vemos que esa organización del entorno de formas muy contrastadas responde a lo que venimos denominando como culturas de aprendizaje. Así, comenzaremos por definir el término "cultura" antropológicamente como "un sistema de representaciones públicas dotadas de significados públicos", según palabras de Sperber (2005: 78). Son representaciones públicas (en el sentido de compartidas) porque solo están conectadas a lo que representan por el significado que les atribuyen aquellos que las producen o utilizan. Por "representaciones culturales", el autor se refiere a aquellas que son muy comunes en un grupo humano. Las representaciones culturales nos ayudan a explicar la construcción colectiva de significados y herramientas que median el aprendizaje.

Para profundizar en las representaciones culturales es necesario explicar por qué algunas de ellas son compartidas por muchas personas. Esta es la idea en la que se basa la epidemiología del autor de las representaciones culturales, es decir, cómo las representaciones son compartidas de manera más o menos generalizada, sin un límite claro entre las representaciones culturales e individuales. Folkestad (2004: 87-88) incide en que "la creación musical tiene lugar en un proceso de interacción entre la experiencia musical de los participantes, sus prácticas culturales, las herramientas, los instrumentos y las instrucciones, que en conjunto forman las competencias en la situación creativa". En esta afirmación podemos ver que tales herramientas, instrumentos e instrucciones tienden a organizarse de forma diferente en función de si se expresan por medio de partituras o de otros tipos de representaciones (por ejemplo, gestos).

Sin establecer asociaciones que simplifiquen los contextos formales e informales, según las herramientas que utilizan, sin embargo, vemos que en cada entorno se tienden a desarrollar y favorecer distintas experiencias y competencias. Por tanto nos interesa adentrarnos en las diferentes formas culturales y sus maneras educativas de transmisión a los miembros que se incorporan a la sociedad. A continuación desarrollamos la idea del continuo entre los polos formal e informal en la educación musical, ampliando los detalles de las descripciones.

\section{LOS CONTEXTOS DE APRENDIZAJE EN LA EDUCACIÓN MUSICAL: DE LOS FORMALES A LOS INFOR- MALES}

Considerando un continuo entre estos dos polos educativos formales e informales se puede encontrar una amplia gama de grises, pero es posible definir los extremos y el punto medio de la siguiente manera. Por una parte, músicos que vienen de una cultura académica que ha dominado la educación en torno a los conservatorios y estructuras regidas por la evaluación, acreditación y distribuidas en cursos. En el punto medio hay un ámbito de la educación, que poco a poco se está incluyendo en la 
educación formal, pero todavía en muchos lugares se encuentra en escuelas privadas. Un ejemplo son las estructuras educativas distribuidas en los cursos y grados, pero con acreditación paralela a la educación formal, por lo que lo denominamos entorno no formal. Y, por último, tenemos un ámbito musical educativo que se define por no graduar en cursos, grados o no certificar oficialmente.

En el caso del contexto informal, la educación musical abarca un sistema social complejo, desde la familia cercana y lejana al alumno, con los profesores como referencia y con un estatus de peso en el grupo social. Esta triple distinción, formal, no formal e informal sirve para describir los diferentes tipos de objetivos, no solo los musicales. Y a su vez establece diferentes tipos de prioridades hacia la música, los parámetros musicales buscados y valorados, y por lo tanto genera diferente impacto en el tipo de prácticas que las diferentes culturas enfatizan (Trilla, 1997). La Tabla 1 muestra las diferencias entre los polos formales e informales, a raíz de Folkestad (2006) y Trilla (1997). 
Tabla 1. Diferencias entre los polos formal

e informal de los contextos de educación musical

\begin{tabular}{|c|c|c|}
\hline & FORMAL & INFORMAL \\
\hline Planificación & Actividad secuenciada a priori & $\begin{array}{l}\text { Actividad no secuenciada a } \\
\text { priori }\end{array}$ \\
\hline Objetivo & $\begin{array}{l}\text { La actividad se focaliza en cómo } \\
\text { aprender a traba- } \\
\text { jar/tocar/componer }\end{array}$ & $\begin{array}{l}\text { La actividad se focaliza en las } \\
\text { maneras de traba- } \\
\text { jar/tocar/componer }\end{array}$ \\
\hline Participantes & $\begin{array}{l}\text { Gestionado por el docente. } \\
\text { Generalmente una persona } \\
\text { lidera la actividad (no necesa- } \\
\text { riamente el profesor en el } \\
\text { sentido formal, pero alguien } \\
\text { dirige y organiza la actividad de } \\
\text { aprendizaje, por ejemplo un } \\
\text { músico del grupo). Esta posición } \\
\text { no tiene por qué ser estática, } \\
\text { aunque usualmente lo es }\end{array}$ & $\begin{array}{l}\text { El proceso parte de la interac- } \\
\text { ción de los participantes en la } \\
\text { actividad }\end{array}$ \\
\hline Motivación & $\begin{array}{l}\text { A veces puede haber conflicto y } \\
\text { diferencias entre las motivacio- } \\
\text { nes del docente y los aprendi- } \\
\text { ces }\end{array}$ & $\begin{array}{l}\text { Se describe como un aprendi- } \\
\text { zaje voluntario y autorregulado }\end{array}$ \\
\hline Lugar & En instituciones & Fuera de instituciones \\
\hline $\begin{array}{l}\text { Estilo de aprendiza- } \\
\text { je (naturaleza y } \\
\text { cualidad del proce- } \\
\text { so) }\end{array}$ & $\begin{array}{l}\text { Aprender a tocar desde la parti- } \\
\text { tura }\end{array}$ & Aprender a tocar de oído \\
\hline $\begin{array}{l}\text { Liderazgo de la } \\
\text { actividad (quién } \\
\text { toma las decisio- } \\
\text { nes) }\end{array}$ & Enseñanza didáctica & $\begin{array}{l}\text { Aprendizaje abierto y autorre- } \\
\text { gulado }\end{array}$ \\
\hline Intencionalidad & Alta & Baja \\
\hline
\end{tabular}

Fuente: Elaboración propia siguiendo a Folkestad (2006) y Trilla (1997).

En los últimos años hemos investigado con culturas de aprendizaje musical representativas de distintos puntos de este continuo formal-informal. Realizamos análisis de contenido del discurso y del tipo de prácticas de músicos de la cultura de aprendizaje del flamenco, que pertenecen a la etnia gitana, y de músicos de la cultura de aprendizaje del jazz y el clásico (Casas-Mas, Pozo y Montero, 2014; Casas-Mas, Montero y Pozo, 2015). Por una parte, la cultura de aprendizaje de música clásica es re- 
presentativa del contexto formal, porque se ubica en instituciones donde la enseñanza está estructurada en cursos, con un proyecto educativo, y donde la acreditación culmina el proceso de evaluación y cumple con los fines sociales de selección.

Por otra parte, las culturas de aprendizaje de música de jazz y flamenco, como músicas populares, muestran distinto grado de formalización que el clásico. La cultura flamenca de aprendizaje, en los contextos en que se mantiene la tradición oral, representa el polo informal, con gran importancia del grupo social y los pares, así como de la transmisión artesanal del conocimiento. Sin embargo, la cultura de aprendizaje del jazz estaría en una posición intermedia que llamamos no formal, por el hecho de utilizar procedimientos o instancias que rompen una o más reglas formales, como la acreditación o el grado de estructuración.

Aplicamos el método lexicométrico, mediante análisis de correspondencias, y encontramos diferencias léxicas significativas entre las tres culturas en las tres dimensiones educativas analizadas (enseñanza, aprendizaje y evaluación). A partir del análisis de sus respuestas completas, finalmente proyectamos una distribución de las tres culturas de aprendizaje en un plano factorial, que resume la distribución de las mismas en función de dos ejes que hemos interpretado en términos de (a) locus de control (autorregulación vs. hetero-regulación) y (b) fenomenología (distancia emocional, analítico, conceptual, conocimiento explícito vs. sensorial, implicación, corporeización, conocimiento implícito).

El discurso de los participantes de la música clásica y los participantes flamencos expresó un aprendizaje hetero-regulado, aunque los participantes clásicos se situaban más cerca de un polo explícito y conceptual, mientras que los participantes flamencos estaban más cerca de un polo implícito, encarnado. El discurso de los participantes del jazz estaba entre los otros dos, más cerca del polo explícito, pero incluyendo un lenguaje característico de autorregulación que no mostró el discurso de las otras dos culturas de aprendizaje.

\section{7. ¿QUÉ APORTA LA COMPARACIÓN DE APRENDIZAJE FORMAL E INFORMAL EN MÚSICA?}

En los estudios descritos en el apartado anterior los participantes conformaban perfiles "puros" de pertenencia a cada una de las tres culturas de aprendizaje. No obstante, nos propusimos analizar también la construcción que hacían de la cultura de aprendizaje del flamenco músicos con un perfil "híbrido" o intercultural (CasasMas, en prensa). Los músicos interculturales en este caso son expertos en el flamenco y además en otra cultura de aprendizaje musical, clásica o jazz. Unos de los participantes provienen de la música clásica y del jazz y actualmente tocan profesionalmente flamenco, mientras que otros tuvieron una inmersión cultural inicial en la música 
flamenca, de familias romaníes, y después se han introducido en el clásico y en el jazz. Todos tocan tanto fusión como sus estilos de origen.

Me concentro en este tipo de músicos porque desarrollan una cierta conciencia de elementos de su propia cultura y de otras debido a los contrastes procedentes de la interacción con diferentes prototipos y formas cotidianas de aprendizaje, enseñanza y concepciones de música. La hibridación facilita el acceso a los procesos de aprendizaje de una manera metacognitiva, es decir, que el aprendiz puede reflexionar sobre sus procesos de pensamiento y la forma en que aprende, propiciando una mayor regulación. Un ejemplo concreto de una descripción literal acerca del aprendizaje musical en el flamenco es este fragmento de un músico de etnia gitana que también toca otras músicas. Él describe cómo la música está fuertemente incluida en la familia, representando los principios tradicionales basados en la oralidad. Define el flamenco como asimilado antes del nacimiento:

"Todo el que está en el flamenco y desde la infancia ha crecido en el flamenco, no sabe por qué, pero tiene algo así como una actitud conservadora; (...) Llevas flamenco dentro (...) El flamenco está aquí [pone la mano sobre el pecho], hay un duende o algún factor que no se aprende. Tú lo absorbes desde la infancia con tu familia».

Son muy relevantes también las aportaciones que hacen sobre el uso de las herramientas de mediación del conocimiento, especialmente de los sistemas de notación y otras representaciones externas. Descrito por el mismo participante: "di que el jazz es más como escribir en papel, se puede organizar todo, pero... no en flamenco". Aquí él alude al chart o esquema melódico-armónico utilizado para escribir los estándares de jazz. O visto por otro músico que proviene del clásico y el jazz:

«En la música clásica puedes limitarte a leer tus piezas; [los flamencos] asimilan el lenguaje hasta el punto de que no tienen que pensar en ello. Aunque no escriben, son más competentes antes que la mayoría de los músicos en entender y desarrollar ideas musicales. A Diego ${ }^{1}$ [Amador] así le sucede. Tiene muchos conceptos dentro pero no sabe que los tiene, y eso lo realza mucho, porque no le crea obstáculos mientras toca».

Estos resultados entroncan con las aportaciones de investigadoras como Green (2001/02), que ha descrito una variedad de prácticas, actitudes y valores en el aprendizaje informal de la música, más centrados en la guitarra de rock y la música popular de la tradición anglo-americana. En sus investigaciones establece que las principales diferencias del aprendizaje informal en comparación con el formal se basan en: el papel de la enculturación en el aprendizaje informal a través de la práctica de la escu-

1 Diego Amador: conocido como el Churri, es un músico sevillano que creció en el barrio de las 3.000 viviendas, un proyecto de conversión del antiguo barrio de etnia gitana, Triana. Considerado uno de los mejores pianistas del flamenco, Amador primero aprendió a tocar la guitarra y también ha tocado otros instrumentos profesionalmente, como la percusión. 
cha con atención y la copia (reproductivo). No solo radica en la disponibilidad de música grabada, sino que se combina con una observación muy cercana que siempre ha formado parte de los paradigmas del folk y de la música tradicional. Esto implica que la práctica y la técnica se adquieren por "ósmosis" en el aprendizaje instrumental con gran énfasis en el desarrollo del "oído".

A menudo, la música que se estudia en el contexto académico difiere de la música que los aprendices escuchan en su día a día, hecho que no sucede en la música popular. En cuanto a la amistad y el aprendizaje entre iguales, en los contextos clásicos occidentales, en ocasiones hay una carencia de hacer música en la comunidad. Mientras, en la música popular, la familia es un ejemplo donde se fomentan actividades en el hogar o en otros lugares donde la gente se encuentra, generalmente con otros fines distintos, pero en los que el hábito de hacer música se convierte en un proceso en el que cualquiera puede participar. Es en estos contextos en los que se promueven la escucha de música en vivo, la imitación y la observación. En otros entornos, la música grabada ha sustituido a la música en vivo y esto ha aumentado la distancia entre músicos y oyentes.

Otros aspectos que Green describe en el aprendizaje informal de la música en oposición al formal son la idea de la experimentación frente al uso práctico de los conocimientos teóricos y la idea de prueba o ensayo frente a la de progresión del contexto formal, de las que se deriva un enfoque diferente para el concepto de "error". Esto también implica una dinámica de mayor riesgo que en la sistematización del aprendizaje formal. Ahora bien, la autora añade que, en los casos en que los músicos de contextos informales se convierten en maestros en contextos formales, no enseñan las mismas estrategias que han aprendido. Ellos asumen la enseñanza tradicional formalizada y enseñan a partir del uso de la notación, sin hacer énfasis en las prácticas de aprendizaje que ellos usaron.

Los ámbitos formales, por otra parte, se enfocan hacia la excelencia técnica y musical. Además, utilizan más habilidades analíticas, seguramente por el uso de la notación. En los ámbitos informales, sin embargo, se hace hincapié en la memorización y la improvisación. En cuanto al tipo de actividades que se priorizan en cada uno, en el formal se prefiere tomar clases y el trabajo en solitario, mientras en el informal se da más importancia a escuchar música de su propio estilo y hacer música solo por diversión con otras personas. Con respecto a la práctica, Green (2001/02) señala que la regularidad y la constancia es una idea crucial en el aprendizaje formal, pero no lo es tanto en el ámbito informal, donde a veces también se considera realizar prácticas mucho más esporádicas e intensas. En la Tabla 2 presentamos una síntesis que refleja las principales ideas defendidas por Green. 
Tabla 2. Síntesis de las diferencias principales entre el contexto formal y el contexto informal descritas por Green

\begin{tabular}{|c|c|c|}
\hline & Formal & Informal \\
\hline $\begin{array}{l}\text { Fuentes de } \\
\text { aprendizaje }\end{array}$ & Desde la partitura & $\begin{array}{l}\text { Observación, copia, e imita- } \\
\text { ción, como por "ósmosis" }\end{array}$ \\
\hline $\begin{array}{l}\text { Música en el día } \\
\text { a día }\end{array}$ & $\begin{array}{l}\text { La música que escuchan en su } \\
\text { día a día difiere con la que } \\
\text { tocan }\end{array}$ & $\begin{array}{l}\text { La música que escuchan en } \\
\text { su día a día coincide con la } \\
\text { que tocan }\end{array}$ \\
\hline Objetivos & $\begin{array}{l}\text { Buscan la excelencia técnica y } \\
\text { musical. Priorizan tomar clases } \\
\text { y el trabajo en solitario }\end{array}$ & $\begin{array}{l}\text { Prefieren hacer música solo } \\
\text { por diversión y escuchar } \\
\text { música de su propio estilo }\end{array}$ \\
\hline $\begin{array}{l}\text { Música en } \\
\text { colectividad }\end{array}$ & $\begin{array}{l}\text { Carencia de hacer música en } \\
\text { comunidad, por amistad con } \\
\text { iguales }\end{array}$ & $\begin{array}{l}\text { Proceso espontáneo en el } \\
\text { que cualquiera del grupo } \\
\text { puede participar. Papel de la } \\
\text { familia }\end{array}$ \\
\hline Audición & $\begin{array}{l}\text { Parámetros moleculares e } \\
\text { independientes. Preponde- } \\
\text { rancia de la altura tonal }\end{array}$ & $\begin{array}{l}\text { Desarrollo del oído global. } \\
\text { Todos los parámetros inte- } \\
\text { grados. Escucha de música } \\
\text { en vivo }\end{array}$ \\
\hline Evaluación & $\begin{array}{l}\text { Evaluación constante de la } \\
\text { propia música y la de otros }\end{array}$ & Menos evaluación \\
\hline \multirow{4}{*}{ Tipo de práctica } & $\begin{array}{l}\text { Uso práctico de los conoci- } \\
\text { mientos teóricos }\end{array}$ & Experimentación \\
\hline & $\begin{array}{l}\text { Progresión. Concepto de } \\
\text { "error" a evitar }\end{array}$ & $\begin{array}{l}\text { Prueba, ensayo-error. } \\
\text { Asumen más riesgos }\end{array}$ \\
\hline & $\begin{array}{l}\text { Enfatizan habilidades analíti- } \\
\text { cas (relacionado con uso de la } \\
\text { notación) }\end{array}$ & $\begin{array}{l}\text { Enfatizan memorización e } \\
\text { improvisación }\end{array}$ \\
\hline & $\begin{array}{l}\text { Sesiones con la idea de regula- } \\
\text { ridad y constancia }\end{array}$ & $\begin{array}{l}\text { Sesiones mucho más esporá- } \\
\text { dicas e intensas }\end{array}$ \\
\hline
\end{tabular}

Fuente: Elaboración propia a partir de Green (2001/02).

La construcción del conocimiento con la ayuda de las diferentes herramientas de mediación dentro de cada cultura de aprendizaje nos permite descifrar toda una gama de colores en un continuo que antes se percibía solo como un blanco o negro, o se reducía a mitos como el "talento" que justificaban si una persona podía acceder o no al aprendizaje musical. 


\section{CONCLUSIONES}

Cerraremos el trabajo con algunas reflexiones, en primer lugar, destacando la variedad de formas de transmisión del conocimiento de las prácticas informales de aprendizaje y de las que los entornos formales podrían aprender tanto (Casas-Mas, 2013; Rogoff, 2012; Scribner y Cole, 1973). Las demandas de la educación formal a menudo están tan desconectadas de las de la vida cotidiana que algunos estudiantes pueden percibir la institución educativa como extraña, hostil y descalificadora de su identidad. De hecho, las escuelas suelen obviar los recursos holísticos, emocionales y encarnados de aprendizaje utilizados en el ámbito musical de la comunidad gitana, al igual que de otras comunidades. En concreto, los parámetros que parecieran más cercanos a esa musicalidad intrínseca del ser humano, los que establecen las redes del vínculo emocional entre los individuos que conforman una comunidad son los que parecen quedar al margen en el ámbito educativo formal.

En segundo lugar, vemos que las herramientas notacionales al servicio de determinado tipo de cultura de aprendizaje, elevadas a un fin en sí mismas parecen dificultar la inclusión de otras culturas de aprendizaje de tradición oral. Así, en consonancia con FornetBetancourt (2004), parece que en el entorno del flamenco la idea de su transcripción y su utilización como herramienta de aprendizaje fuera sinónimo de "objetivación" y tuviera graves repercusiones en su forma originaria de sonorizar el mundo. Se percibe la sensación de alterar su cosmovisión puesto que esa sonorización cotidiana y colectiva construye su identidad (como sucedió en los orígenes del jazz y otras músicas populares). Es necesario repensar el lenguaje musical escrito para que no encasille o al menos considere cómo anotar la variedad de estructuras rítmicas, tímbricas y de afinación de músicas basadas en la oralidad. Pero no se trata sólo de la objetivación de esos parámetros técnicos, también del contexto comunicativo y social al que está vinculado cada canto, cada pieza musical.

Un verdadero proyecto intercultural en la institución académica tendría que contemplar la posibilidad y la exigencia de ampliar el conocimiento de las músicas populares y de tradición oral, recuperándolas desde su oralidad, sin que el aprendizaje de la escritura tenga que ser una exigencia para ello (Rodríguez, 2009). Las múltiples herramientas notacionales no dejan de ser eso, herramientas, y como tal son elementos que han dado pie a conocimiento muy valioso en la humanidad. No obstante, si se convierten en un fin en sí mismas se propicia su uso reproductivo en lugar de un uso epistémico, o de transformación del propio conocimiento y del colectivo. Este cambio de concepción parte de asimilarlas en importancia y fomentar la recuperación de otras representaciones externas más ambiguas como el gesto y las basadas en la audición. Repensar los hábitos de comunicación y escucha a partir de prácticas que generen la alteración de los mismos (Hormigos, 2010) son los medios que tiene el contexto académico a su disposición para superar la crisis de los cánones estéticos tradicionales y de la noción misma de obra de arte. 


\section{BiBLIOGRAFÍA}

BLACKING, J. [1976] (2006): ¿Hay música en el hombre? Madrid: Alianza.

CASAS-MAS, A. (2013). Las culturas de aprendizaje musical: concepciones, procesos y prácticas de aprendizaje en clásico, flamenco y jazz. Madrid: Universidad Autónoma de Madrid. Tesis doctoral.

CASAS-MAS, A. (en prensa): "Developing an approach to the flamenco teaching-learning culture: an innovative (traditional) learning", en Leung, B. W. (ed.): Traditional musics in the modern world. Springer.

CASAS-MAS, A., MONTERO, I. y POZO, J. I. (2015): “El discurso sobre la práctica de un guitarrista de jazz semiprofesional. Estudio de caso de aprendizaje musical constructivo", Journal of the International Association for the Study of Popular Music, vol. 5, no 1, pp. 54-80. DOI: http://dx.doi.org/10.5429/2079-3871(2015)v5i1.5es

CASAS, A. y POZO, J. I. (2008): “¿Cómo se utilizan las partituras en la enseñanza y aprendizaje de la música?”, Cultura y Educación, vol. 20, no 1, pp. 49-62. DOI: http://dx.doi.org/10.1174/113564008783781503

CASAS-MAS, A., POZO, J. I. y MONTERO, I. (2014): "The influence of music learning cultures on the construction of teaching-learning conceptions", British Journal of Music Education, vol. 31, no 3, pp. 319-342. DOI: http://dx.doi.org/10.1017/S0265051714000096

CASAS-MAS, A., POZO, J. I. y SCHEUER, N. (2015): "Musical learning and teaching conceptions as sociocultural productions in classical, flamenco, and jazz cultures", Journal of Cross-Cultural Psychology, vol. 46, no 9, pp. 1.191-1.225. DOI: http://dx.doi.org/10.1177/0022022115603124

DUNBAR-HALL, P. y WEMYSS, K. (2000): "The effects of the study of popular music on music education", International Journal of Music Education, vol. 36, no 1, pp. 23-34.

FOLKESTAD, G. (1998): "Musical learning as a cultural practice. As exemplified in computerbased creative music making", en Sundin, B., McPherson, G. y Folkestad, G. (eds.): Children Composing. Malmö: Lund University / Malmö Academy of Music.

FOLKESTAD, G. (2004). "A meta-analytic approach to qualitative studies in music education: A new model applied to creativity and composition", Bulletin of the Council for Research in Music Education, no 161-162, pp. 83-90.

FOLKESTAD, G. (2006). "Formal and informal learning situations or practices vs formal and informal ways of learning", British Journal of Music Education, vol. 23, no 2, pp. 135-145.

FORNET-BETANCOURT, R. (2004): Sobre el concepto de interculturalidad. México: Consorcio Intercultural.

GREEN, L. (2001/02): How popular musicians learn. A way ahead for music education. Aldershot: Ashgate. 
GREEN, L. (2008): Music, informal learning and the school: A new classroom pedagogy. Aldershot: Ashgate.

GRUSON, L. M. (1988). "Rehearsal Skill and musical competence: does practice make perfect?", en Sloboda, J. A. (ed.): Generative processes in music: the psychology of performance, improvisation, and composition. New York: Oxford University, pp. 91-112.

HALLAM, S. (2007): Music psychology in education. London: University of London.

HORMIGOS, J. (2010): “Distribución musical en la sociedad de consumo: la creación de identidades culturales a través del sonido", Comunicar, vol. XVII, p. 34, pp. 91-98. DOI: https://doi.org/10.3916/C34-2010-02-09

HULTBERG, C. (2002): "Approaches to music notation: the printed score as a mediator of meaning in western tonal tradition", Music Education Research, vol. 4, n으 2, 185-197.

KINGSBURY, H. (1988): Music, talent and performance: A conservatory cultural system. Filadelfia: Temple University.

LILLIESTAM, L. (1996): “On playing by ear”, Popular Music, vol. 15, no 2, pp. 195-216.

MCPHERSON, G. E. y GABRIELSSON, A. (2002): "From sound to sign", en Parncutt, R. y McPherson, G. E. (eds.): The science and psychology of musical performance: Creative strategies for music teaching and learning. Oxford: Oxford University, pp. 99-115.

MONTERO, I. (1989): Motivación de logro. Concepto y medida en el ámbito de la enseñanza media. Madrid: Universidad Autónoma de Madrid. Tesis doctoral no publicada.

MUÑOZ CARRIÓN, A. (2008): "El patrimonio cultural material y el inmaterial: buenas prácticas para su preservación", Mediaciones Sociales, no 3, pp. 495-534. Disponible en https://goo.gl/poF92n. Consultado el 17 de agosto de 2016.

MUSUMECI, O. (2002): "Hacia una educación de conservatorio humanamente compatible", en VV.AA.: Actas de la Segunda Reunión Anual de SACCoM. Quilmes. Disponible en https://goo.gl/QrCMI7. Consultado 3 de junio de 2016.

NETTL, B. (1995): Heartland excursions: ethnomusicologial reflections on schools of music. Illinois: University of Illinois.

ROBINSON, T. (2010): How popular musicians teach. Sheffield: University of Sheffield. Tesis doctoral no publicada.

RODRÍGUEZ, E. (2009): "Reflexiones en torno al mestizaje y sus implicaciones para la relación oralidad/escritura en el marco de la discusión intercultural", Revista Interamericana de Educación de Adultos, vol. 31, no 2, pp. 187-198.

ROGOFF, B. (2012): "Learning without lessons: opportunities to expand knowledge", Infancia y Aprendizaje, vol. 35, no 2, pp. 233-252. DOI: http://dx.doi.org/10.1174/021037012800217970

SCRIBNER, S. y COLE, M. (1973): "Cognitive consequences of formal and informal education", Science, vol. 182, pp. 553-559. 
SHAH, S. M. (2006): "Popular music in Malaysia: education from the outsider", International Journal of Music Education, vol. 24, no 2, pp. 132-139.

SPERBER, D. (2005): Explicar la cultura. Un enfoque naturalista. Madrid: Morata.

TRILLA, J. (1997): "Relaciones entre la educación formal, la no formal y la informal", en La educación fuera de la escuela. México: Ariel, pp. 187-196.

UNESCO (2010): "Convention for the Safeguarding of the Intangible Cultural Heritage. Nomination file no. 00363". Disponible en https://goo.gl/e9i6Rj. Consultado el 1 de abril de 2016.

VALLEJO, P. (2008): Patrimonio musical de los Wagogo (Tanzania): contexto y sistemática. Madrid: Cyan.

VYGOTSKI, L. S. [1931] (2000): Obras escogidas III: Historia del desarrollo de las funciones psíquicas superiores. Madrid: Aprendizaje Visor.

VYGOTSKI, L. S. y LURIA, A. R. [1930] (1993): Studies on the history of behaviaour: ape, primitive and child. Hillsdale, New Jersey: Lawrence Erlbaum.

WANG, J. C. y HUMPHREYS, J. T. (2009): "Multicultural and popular music content in an American music teacher education program", International Journal of Music Education, vol. 27, no 1 , pp. 19-36.

WERTSCH, J. V. [1991] (1993): Voces de la mente. Un enfoque sociocultural para el estudio de la acción mediada. Madrid: Visor.

WOODY, R. H. (1999): "The relationship between explicit planning and expressive performance of dynamic variations in an aural modelling task", Journal of Research in Music Education, vol. 47, no 4, 331-342.

Amalia CASAS-MAS es Profesora Superior de Piano, y de Lenguaje Musical, Teoría Musical, Transposición y Acompañamiento. Es licenciada y doctora en Psicología por la Universidad Autónoma de Madrid. Ha sido docente durante 12 años en el Real Conservatorio Superior de Música de Madrid, y otros centros de Enseñanzas Artísticas Superiores del país. Actualmente es profesora en la Universidad Autónoma de Madrid, impartiendo docencia en la Facultad de Formación de Profesorado. Sus líneas de investigación se centran en las diferentes formas de aprendizaje de la música (concepciones, procesos psicológicos, sistemas externos de representación y prácticas) desde contextos formales a informales, especializada en las comunidades de flamenco de etnia gitana. Es socia fundadora de la recientemente constituida Asociación Española de Psicología de la Música y la Interpretación Musical (AEPMIM). Correo electrónico: amalia.casas@uam.es. 